\title{
Performance Evaluation of Connection Admission Control for IEEE 802.16 Networks
}

\author{
Shwetha.D \\ Dept. of Electronic Science \\ Bangalore University \\ Bangalore -560056
}

\author{
Subramanya Bhat.M \\ Dept. of Electronic Science \\ Bangalore University \\ Bangalore -560056
}

\author{
Devaraju.J.T \\ Dept. of Electronic Science \\ Bangalore University \\ Bangalore -560056
}

\begin{abstract}
Quality of Service (QoS) provisioning to the various kinds of network traffic is one of the major design criteria of IEEE 802.16 WiMAX standard. The MAC and physical layers of 802.16 standards are designed to support different types of real time application by providing QoS. Scheduling, Connection Admission Control (CAC) and traffic policing are the major issues to ensure QoS. In standard, scheduling and admission control are kept as open issues. Admission control is the ability of a network to control admission of new traffic based on the availability of resources. As per the specification the CAC considers minimum reserved rate of a connection as an admission criterion, in which the system can admit more connections, but packets of admitted connection may encounter large delays. In this paper average data rate (avg-rate CAC) and maximum sustained rate (max-rate $\mathrm{CAC}$ ) of the connections are considered as admission criteria in $\mathrm{CAC}$, along with minimum reserved rate (min-rate $\mathrm{CAC}$ ). The performance of the WiMAX network is evaluated and compared for min-rate, avg-rate and max-rate $\mathrm{CAC}$ by considering the performance metrics such as number of connections admitted, throughput and delay using QualNet simulation tool.
\end{abstract}

\section{Keywords}

WiMAX, Scheduling, Connection Admission Control \& Quality of Service

\section{INTRODUCTION}

The IEEE 802.16 standard also known as WiMAX has been ratified by IEEE as a Wireless Metropolitan Area Network (WMAN) technology [1-2]. The mobility concept is introduced in 802.16e standard which defines Air Interface for Mobile Broadband Wireless Access (BWA) systems and designed to support seamless handover while maintaining differentiated Quality of Service (QoS) [1-2]. The standard supports QoS by classifying services into different service types. The service types in $802.16 \mathrm{e}$ have been designed to support real time applications like voice, video and non-real time application like file transfer. The service types defined are Unsolicited Grant Service (UGS), Extended Real-time Polling Service (ertPS), Real-time Polling Service (rtPS), Non-Real-time Polling Service (nrtPS) and Best Effort (BE). The specifications for these service types such as minimum reserved rate, maximum sustained rate, maximum latency, tolerated jitter, traffic priority etc are defined in the standard, but the standard does not specify any scheduling architecture.

In addition to scheduling, Connection Admission Control (CAC) is also an important aspect in providing QoS guarantee for
WiMAX network. CAC concerns about how to minimize the blocking of connection requests and the QoS violation due to many admitted connections [3]. CAC admits the new connection only if such admission will not compromise the performance of existing traffic. Whenever a new connection request comes, based on availability of resources CAC will admit or reject the connection. New connections which seek for the admission will be of two types, they are handoff sessions and newly originated connections. Handoff sessions are often given higher priority over new sessions from an admission control standpoint.

As per the specification the CAC needs to consider minimum reserved rate of a connection as an admission criterion [1]. If the available bandwidth is more than the minimum reserved rate, then that connection will be admitted, otherwise will be rejected. In this paper along with minimum reserved rate, average data rate and maximum sustained rate of the connections are also considered as admission criteria for CAC. The performance of the WiMAX network is evaluated for these three CAC admission criteria using QualNet simulation tool [4]. The rest of this paper is organized as follows. Section 2 outlines the related work in the literature. Section 3 and 4 describe the overview of WiMAX network and three CAC mechanisms respectively. Section 5 presents simulation results followed by conclusion in section 6.

\section{RELATED WORK}

As the CAC is one of the major issues in providing QoS for WiMAX network, many researchers have proposed the algorithms for CAC. Authors of [5-6] have proposed adaptive hierarchical polling approach with cost-based CAC and Markov decision process based $\mathrm{CAC}$ respectively for increasing utilization of access channel and network reward and reducing polling delay. $\mathrm{H}$ Wang et.al [7] proposed a CAC algorithm which provides the highest priority for UGS flows and maximizes the bandwidth utilization by bandwidth borrowing and degradation. Jinchang $\mathrm{Lu}$ et.al [8] proposed a cross-layer elastic CAC and holistic opportunistic scheduling for point-tomultipoint (PMP) networks. In order to avoid the QoS degradation authors of [9] have proposed a statistical CAC mechanism which considers the traffic variability and overflow. Authors of [10-11] have proposed CAC algorithms which are based on bandwidth estimation of connections. The authors of [12] have proposed a CAC that improves the QoS of BE traffic by avoiding a strict bandwidth assignment of other traffics (rtPS and nrtPS). The authors of [13] have used Markov Chain model for the integration of CAC and uplink packet scheduling (UPS) mechanism to identify quantitative measurement of some QoS parameters like delay, loss rate, throughput, connection 
acceptance probabilities and bandwidth utilization of the system. The authors of [14-15] used the concept of adaptive bandwidth degradation to satisfy both bandwidth and delay requirements of the admitted connections and to improve the bandwidth utilization (BU) of the system. In [15] priority is given to handoff connections. Tzu-Chieh Tsai et.al [16] proposed an UPS with CAC mechanism that is token bucket based. In [17] a new metric called interference ratio (IR) is employed as the criteria of degradation, which describes the new connection request's interference to QoS of degraded existing services. The authors of [18] proposed a token-bucket based UPS combined with CAC of 802.16 and presented a token bucket based traffic flows model. In paper [19] the authors proposed conservative and nonconservative CAC schemes along with bandwidth allocation method for WiMAX system. I. Ahmad et.al [20] proposed a CAC scheme that estimates the usable link capacity for WiMAX communication at various vehicular speeds and uses this information while making a CAC decision. In paper [21] D. S. Shu'aibu et.al proposed a CAC scheme with dual partitioning in which, the total link bandwidth is divided into two partitions, one partition is dedicated to all traffics which have variable bit rate and the second portion is dedicated to constant bit rate traffics, based on this partition CAC algorithm was developed. C. Tarhini et.al [22] proposed a density-based CAC where the degree of acceptance of flows depends on the density of the users in the given location. In paper [23] authors proposed a combined link aware CAC with dual partitioning and packet scheduling for BE and UGS traffics to achieve high throughput with maximum link utilization.

\section{OVERVIEW OF WIMAX NETWORK}

IEEE 802.16 (WiMAX) standard provides specification for medium access control (MAC) layer and physical (PHY) layer of combined fixed and mobile BWA systems providing multiple services. PHY layer uses adaptive modulation and coding (AMC) schemes combined with orthogonal frequency division multiple access (OFDMA) to produce variable data rates to support variable channel conditions. A typical 802.16 network consists of a base station (BS) and a number of subscriber stations (SS) which communicate with the BS. Communication can happen in two modes: PMP mode and mesh mode. In mesh mode SSs can communicate with each other without the need of BS. In PMP mode, all communications happen through the BS and the BS acts as the central entity that decides the transmission and reception schedule of the SSs. The communication path between SS and BS has two directions: uplink (UL: from SS to BS) and downlink (DL: from BS to SS), multiplexed either with time division duplex (TDD) or frequency division duplex (FDD).

Our study is based on the PMP mode with TDD. Each TDD frame is divided into a DL subframe and an UL subframe. Each subframe consists of an integer number of physical slots (PSs), which represents the minimum unit of bandwidth allocation. Each frame contains DL-MAP and UL-MAP, which describe the usage of PSs in DL and UL directions respectively. Each SS receives and decodes the DL-MAP and looks for MAC headers indicating data for itself in the remainder of the DL subframe. Through the UL-MAP, the BS informs the transmission opportunities of SSs (which PSs of the uplink subframe it is allowed to transmit in), based on the bandwidth requests made by each SS. SS uses bandwidth request mechanisms to specify uplink bandwidth requirement to the BS. The BS will grant the bandwidth to SS in two modes. They are (i) Grant Per Connection (GPC): Each connection is treated separately and bandwidth is allocated to each connection explicitly. SS then transmits in the order specified by the BS. (ii) Grant Per Subscriber Station (GPSS): All connections from a single SS are treated as single unit and bandwidth is granted accordingly by the BS on a per SS basis. An additional scheduler in the SS determines the service order among its connections in the granted slots. Transmission parameters, including the modulation parameters and coding schemes, may be adjusted individually for each SS on a frame-by-frame basis [24].

The MAC layer of 802.16 standard is connection oriented i.e., all data communications, for both transport and control, are in

Table 1. Details of QoS classes

\begin{tabular}{|c|c|c|}
\hline QoS Service Type & QoS specifications & Applications \\
\hline Unsolicited Grant Service (UGS) & $\begin{array}{c}\text { Minimum reserved rate, Maximum } \\
\text { sustained rate, Traffic priority, } \\
\text { Maximum latency tolerance }\end{array}$ & T1/E1 transport \\
\hline $\begin{array}{c}\text { Extended Real-time Polling } \\
\text { Service (ertPS) }\end{array}$ & $\begin{array}{c}\text { Minimum reserved rate, } \\
\text { Maximum sustained rate, } \\
\text { Traffic priority, Maximum latency } \\
\text { tolerance, Jitter tolerance }\end{array}$ & VoIP \\
\hline $\begin{array}{c}\text { Real-time Polling Service (rtPS) } \\
\text { Minimum reserved rate, Maximum } \\
\text { sustained rate, Traffic priority, Maximum } \\
\text { latency tolerance }\end{array}$ & MPEG Video \\
\hline $\begin{array}{c}\text { Non-real-time Polling Service } \\
\text { (nrtPS) }\end{array}$ & $\begin{array}{c}\text { Minimum reserved rate, Maximum } \\
\text { sustained rate, Traffic priority }\end{array}$ & $\begin{array}{c}\text { FTP with guaranteed } \\
\text { minimum throughput }\end{array}$ \\
\hline Best Effort (BE) & Maximum sustained rate, Traffic priority & HTTP \\
\hline
\end{tabular}


the context of a unidirectional connection. Each packet has to be associated with a connection at MAC level. Each connection is assigned a unique connection identifier (CID) and a service flow identifier (SFID) with an associated service class. The SS cannot transmit data until it has been allocated a channel by the BS. This provides a way for bandwidth request, association of QoS and other traffic parameters and data transfer related actions. This allows 802.16e to provide strong support for QoS. QoS in $802.16 \mathrm{e}$ is supported by allocating each connection between the SS and the BS (called a service flow in 802.16 terminologies) to a specific QoS class (service type). In 802.16e, there are 5 QoS classes; the Table 1 gives the details of QoS classes.

\section{CONNECTION ADMISSION CONTROL MECHANISM}

Connection admission control (CAC) is the ability of a network to control admission to new traffic, based on resource availability. When a new connection is generated or parameters of ongoing connection are updated at the SS, then SS sends a message to the BS through dynamic service addition/change/delete (DSA/DSC/DSD) requests. The classifier at the BS depending on the type of service request, classifies it into one of the priority queues and $\mathrm{BE}$ requests do not go through CAC process. The priority queues are accessed by the $\mathrm{CAC}$ module in order to check whether the requested QoS can be guaranteed in the current situation at the BS. If accepted, each connection will be allotted a unique CID and the CAC informs the scheduler to allocate bandwidth request slots in the next scheduling interval to that connection. If the connection is accepted by CAC, the SS will then send its bandwidth request (for non-UGS connections) which will be classified and directed to the appropriate priority queue on the basis of CID [24].

As per the specification the $\mathrm{CAC}$ considers minimum reserved rate of a connection as an admission criterion (min-rate CAC) [1]. If the available bandwidth is more than the minimum reserved rate, then that connection will be admitted, otherwise will be rejected. The available bandwidth after admitting $\boldsymbol{n}$ connections is given by

$$
\mathrm{BW}_{\text {available }}=\mathrm{BW} \text { total }-\sum_{\mathrm{i}=1}^{\mathrm{n}} \mathrm{R}_{\mathrm{i}}
$$

Where $\mathrm{BW}_{\text {total }}$ is the total link bandwidth and $\mathrm{R}_{\mathrm{i}}$ is the minimum reserved rate of $i^{\text {th }}$ connection. A new connection request $\boldsymbol{k}$ with minimum reserved rate $R_{k}$ will be admitted only if

$$
\mathrm{BW}_{\text {available }}>\mathrm{R}_{\mathrm{k}}
$$

BS updates the available bandwidth after admitting the connections. CAC can admit more connections if it considers only minimum reserved rate as admission criterion, but packets of admitted connection may encounter large delays [11].

In this paper CAC considers average data rate (avg-rate CAC) and maximum sustained rate (max-rate CAC) of the connections as admission criteria, along with minimum reserved rate (minrate $\mathrm{CAC}$ ).

\subsection{Avg-rate CAC}

In avg-rate $\mathrm{CAC}$, the $\mathrm{CAC}$ considers average of minimum reserved rate and maximum sustained rate of a connection as an admission criterion. If the available bandwidth is more than the average rate of the connection, then that connection will be admitted, otherwise will be rejected.

\subsection{Max-rate CAC}

In max-rate $\mathrm{CAC}$, the $\mathrm{CAC}$ considers maximum sustained rate of a connection as an admission criterion. If the available bandwidth is more than the maximum sustained rate of the connection, then that connection will be admitted, otherwise will be rejected.

\section{SIMULATION AND RESULTS}

The performances of min-rate CAC, avg-rate CAC and max-rate CAC are evaluated using QualNet 5.0.2 simulator and their performances are compared. While the evaluation, only UGS, ertPS, rtPS and nrtPS traffic classes are considered, BE traffic is not considered since it does not go through admission control. A single WiMAX cell is considered in the simulation area of $1 \mathrm{Km}$ x $1 \mathrm{Km}$ working at a frequency $2.4 \mathrm{GHz}$. The path loss model selected is two-ray with constant shadowing model of shadowing mean $4 \mathrm{~dB}$. The simulation parameters settings are mentioned in Table 2.

Table 2. Simulation parameters

\begin{tabular}{|c|c|}
\hline Property & Value \\
\hline Simulation time & $45 \mathrm{Sec}$ \\
\hline Channel bandwidth & $20 \mathrm{MHz}$ \\
\hline FFT size & 2048 \\
\hline Antenna model & Omni directional \\
\hline BS antenna gain & $10 \mathrm{dBi}$ \\
\hline SS antenna gain & $0 \mathrm{dBi}$ \\
\hline BS antenna height & $12 \mathrm{~m}$ \\
\hline SS antenna height & $1.5 \mathrm{~m}$ \\
\hline
\end{tabular}

\subsection{Scenario 1}

In this scenario a single BS and eight SSs are considered. This scenario is designed to study the behaviour of CAC mechanism with the increase in number of connection. The number of connections is increased from 4 (UGS, ertPS, rtPS and nrtPS connections of one each) to 32 insteps of 4 (connections of each service type are increased by one) with the data rate of $2 \mathrm{Mbps}$ for each connection. The performances of three CAC mechanisms are evaluated by considering metrics such as number of connections admitted by the CAC, throughput and average end-to-end delay of connections.

Figure 1 gives the plot of number of connections admitted for min-rate, avg-rate and max-rate CAC mechanisms with respect to increase in number of connections. For lesser connections (upto 8 connections) the number of connections admitted is same for all the three CAC mechanisms. As the number of connections increases, the min-rate $\mathrm{CAC}$ out performs the other two $\mathrm{CAC}$ mechanisms, since the min-rate $\mathrm{CAC}$ is required to guarantee only minimum reserved rate [11]. The max-rate $\mathrm{CAC}$ admits lesser connections as it is required to guarantee maximum sustained rate to each connection and avg-rate CAC admits more connections compared to max-rate CAC. 


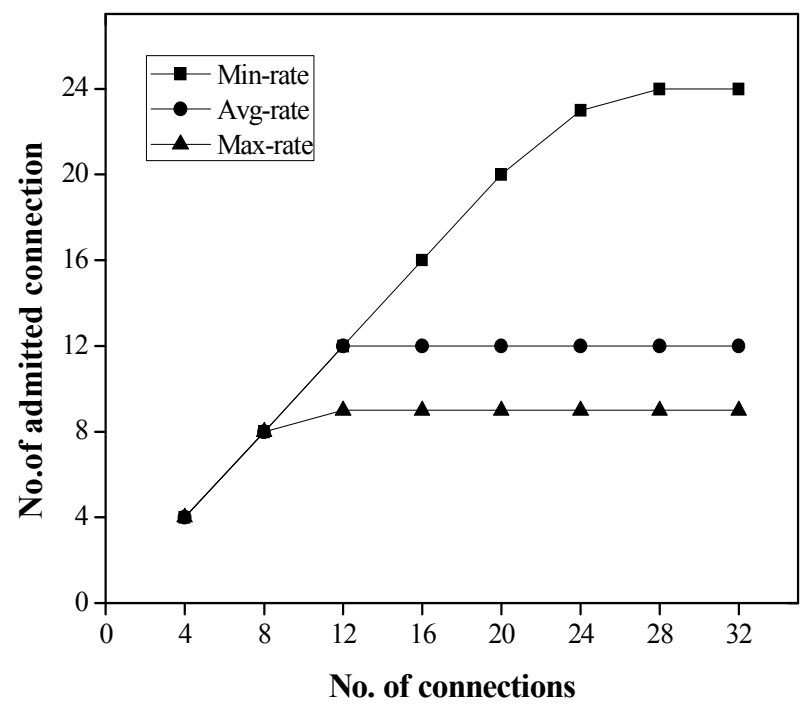

Fig 1: Number of connections admitted for varying number of connections

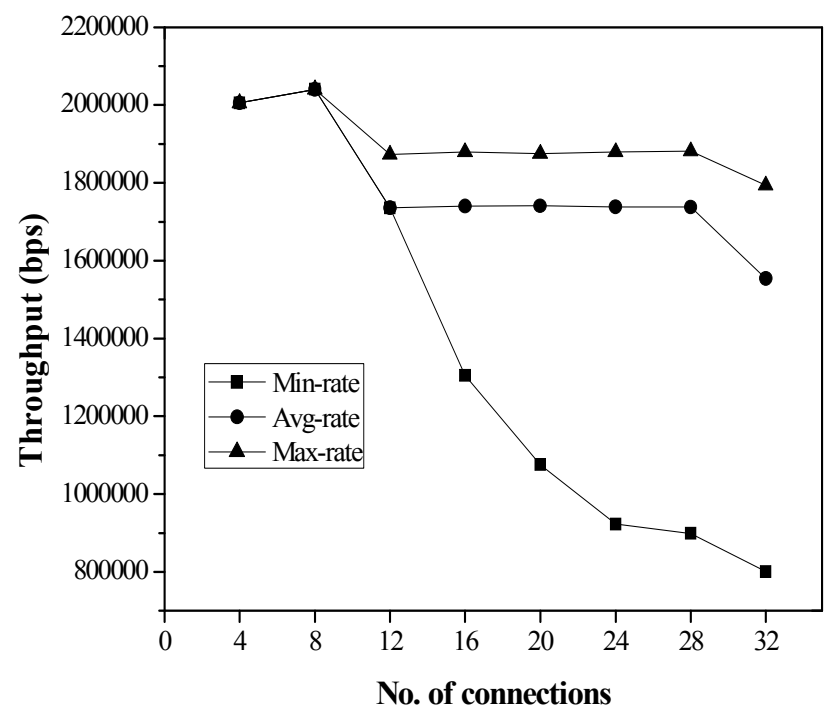

Fig 2: Throughput performances for varying number of connections

The throughput and delay performances for increase in number of connections are shown in Figure $2 \& 3$ respectively. The throughput and delay performances of max-rate CAC are good compared to min-rate and avg-rate CAC, since admitted connections by max-rate $\mathrm{CAC}$ are guaranteed with maximum sustained rate. The min-rate CAC has poor throughput performance, as it guarantees only minimum reserved rate to each connection and the packets encounters larger delays compared to other two CACs, since number of admitted connections is more. As avg-rate CAC admits optimum number of connections by giving avg-rate to each connection, the throughput and delay performances are better compared to minrate CAC.

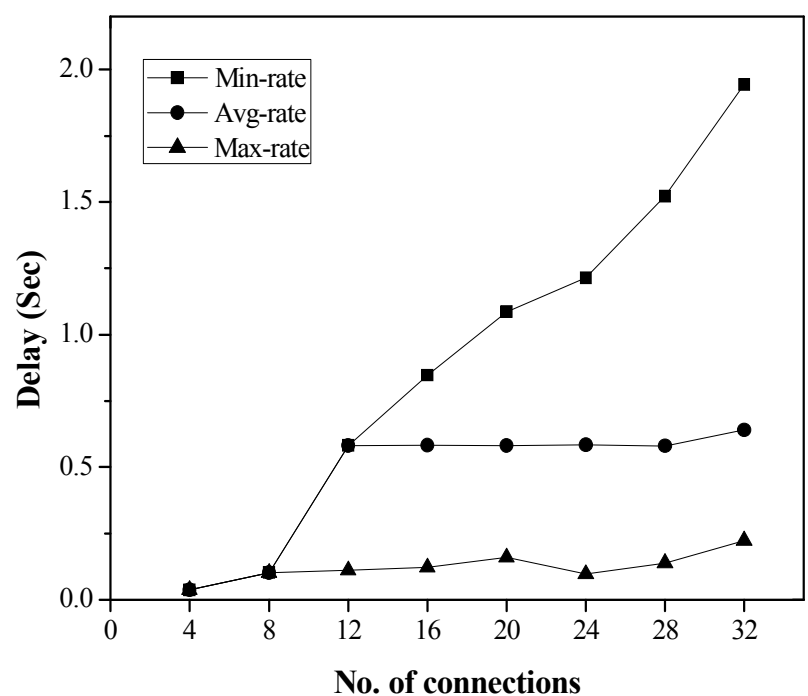

Fig 3: Delay performances for varying number of connections

\subsection{Scenario 2}

In this scenario all the parameters of scenario 1 are retained and number of connections is kept constant at 12 (UGS, ertPS, rtPS and nrtPS connections of three each) and 20 (UGS, ertPS, rtPS and nrtPS connections of five each). The system load is increased by increasing the data rate of each connection. The data rate is increased from $1 \mathrm{Mbps}$ to $5 \mathrm{Mbps}$. Performances of avg-rate $\mathrm{CAC}$ and max-rate $\mathrm{CAC}$ are compared with min-rate CAC by considering the number of connections admitted, throughput and delay performances.

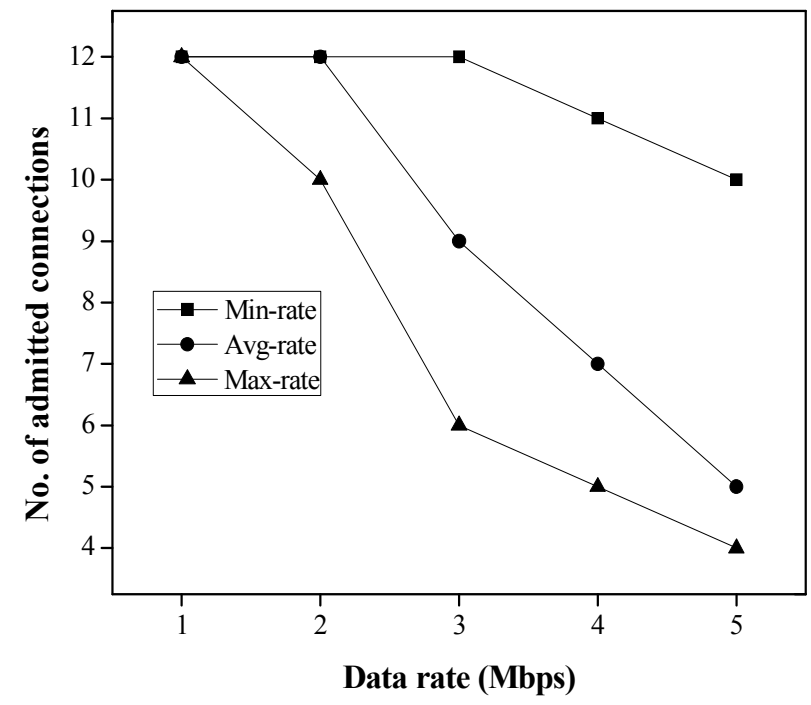

Fig 4: Number of connections admitted for varying data rate for 12 connections 


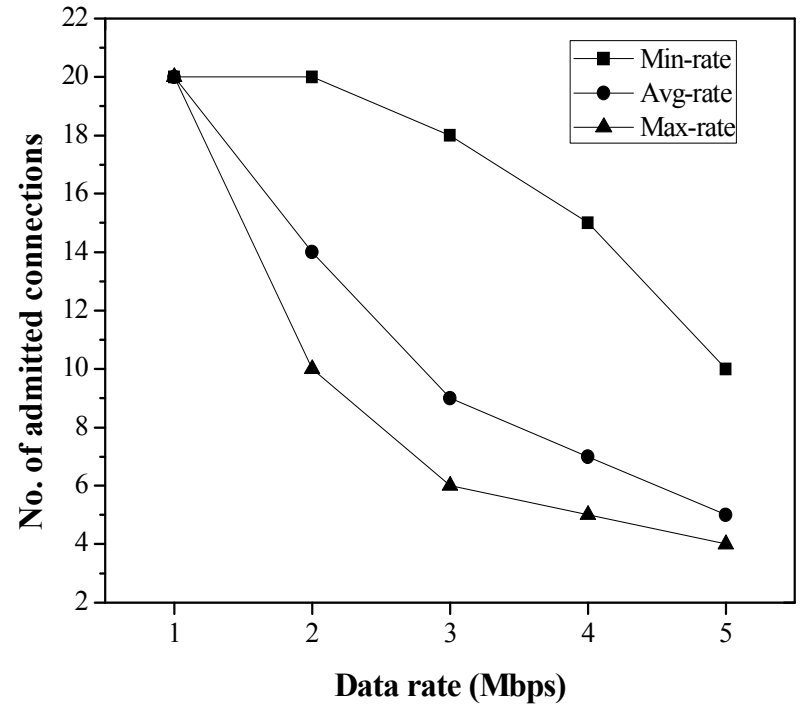

Fig 5: Number of connections admitted for varying data rate for 20 connections

Figure $4 \& 5$ gives the number of connections admitted for varying data rate for 12 and 20 connections respectively. As the data rate increases the number of connections that can be admitted by all the three CACs decreases, since the bandwidth requirement of each connection increases. As the min-rate CAC is required to guarantee only minimum reserved rate, the number of connections admitted is more compared to avg-rate and maxrate. The max-rate $\mathrm{CAC}$ admits lesser connections as it is required to guarantee maximum sustained rate to each connection. As the data rate increases the number of connections admitted for both 12 and 20 connections becomes same.

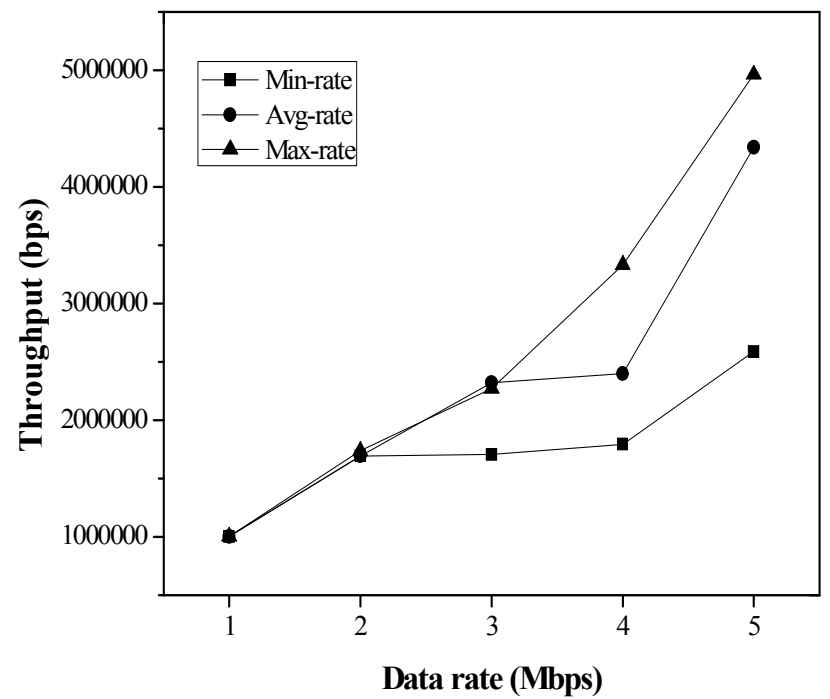

Fig 6: Throughput performances for varying data rate for 12 connections

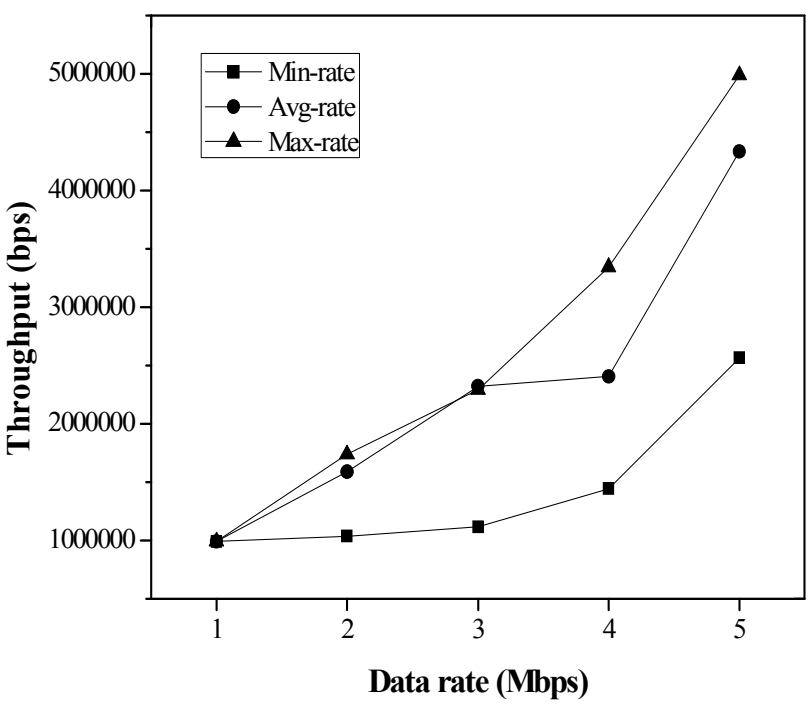

Fig 7: Throughput performances for varying data rate for 20 connections

The throughput performances for varying data rate for 12 and 20 connections are shown in Figure $6 \& 7$ respectively. As the data rate increases, throughput of connections increases for both 12 and 20 connections. The throughput performance of max-rate CAC is good compared to other two CAC mechanisms. As the avg-rate CAC admits optimum number of connections, it has better throughput performances compared to min-rate CAC.

The delay performances for varying data rate are given in Figure 8 \& 9 for 12 and 20 connections respectively. As the admitted connections by max-rate are guaranteed with maximum sustained rate, the delay performance is better compared to avgrate and min-rate $\mathrm{CAC}$.

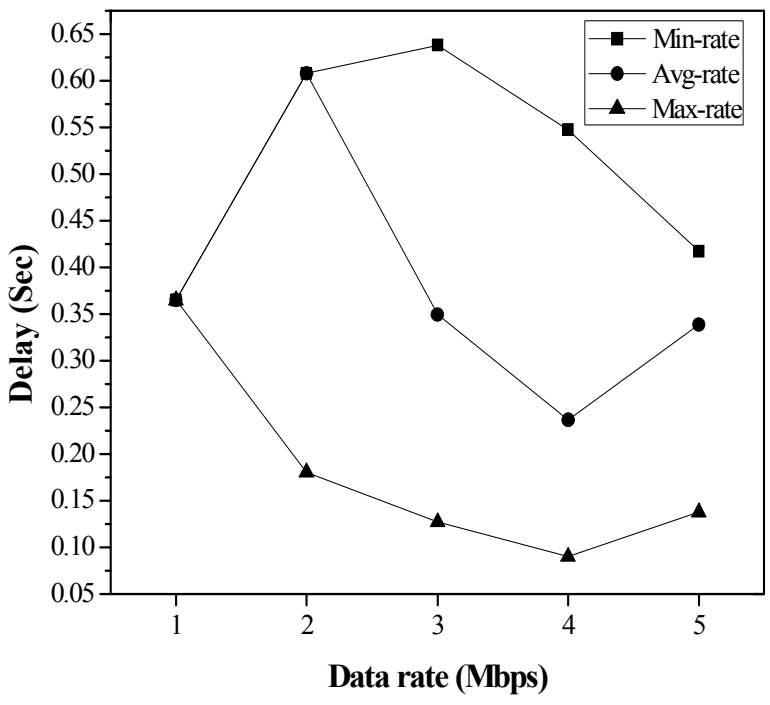

Fig 8: Delay performances for varying data rate for 12 connections 


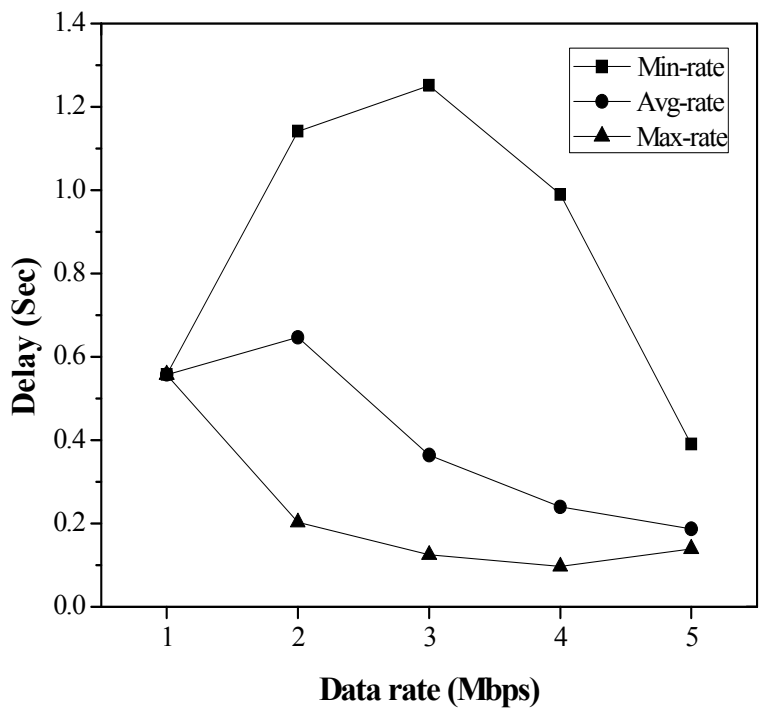

Fig 9: Delay performances for varying data rate for $\mathbf{2 0}$ connections

\section{CONCLUSION}

In this paper the performance of WiMAX network is evaluated for min-rate, avg-rate and max-rate $\mathrm{CAC}$ mechanisms which consider minimum reserved rate, average data rate and maximum sustained data rate as the admission criteria respectively. The performances of avg-rate and max-rate CAC are compared with the min-rate CAC which is specified in the standard. The simulation results show that the delay and throughput performances of max-rate CAC are good but it admits lesser connections. The throughput and delay performances of avg-rate CAC are better compared to min-rate $\mathrm{CAC}$ and number of connections admitted is more than that of max-rate CAC.

\section{ACKNOWLEDGMENTS}

The authors would like to thank UGC for sanctioning the funds under major research project.

Authors would also thank Nihon communication, Bangalore for EXata tool.

\section{REFERENCES}

[1] IEEE802.16e: IEEE Standard for Local and metropolitan area networks Part 16: Air Interface for Fixed and Mobile Broadband Wireless Access Systems, 2005.

[2] IEEE802.16e: IEEE Standard for Local and metropolitan area networks Part 16: Air Interface for Broadband Wireless Access Systems, 2009.

[3] Byeong Gi Lee and Sunghyun Choi, 2008. Broadband Wireless Access and Local Networks - Mobile WiMAX and WiFi, Artech House.

[4] Documentation of QualNet simulation tool, http://www.scalablenetworks.com $\backslash$ qualnet/documentation

[5] Ben-Jye Chang, Yan-Ling Chen and Chien-Ming Chou, 2007. "Adaptive Hierarchical Polling and Cost-based Call
Admission Control in IEEE 802.16 WiMAX Networks", In proceedings of IEEE WCNC 2007, 2757-2761.

[6] Ben-Jye Chang and Yan-Ling Chen, 2008. "Adaptive Hierarchical Polling and Markov Decision Process Based CAC for Increasing Network Reward and Reducing Average Delay in IEEE 802.16 WiMAX Networks", Computer Communications 31, 2280-2292.

[7] Haitang Wang, Wei Li and Dharma P. Agrawal, 2005. "Dynamic Admission Control and QoS for 802.16 Wireless MAN," Wireless Telecommunications Symposium, 60-66.

[8] Jinchang Lu and Maode Ma, 2010. "A Cross-layer Elastic CAC and Holistic Opportunistic Scheduling for QoS Support in WiMAX", Computer Networks 54, 1155-1168

[9] Ke Yu, Xuan Wang, Songlin Sun, Lin Zhang and Xiaofei $\mathrm{Wu}, 2009$. "A Statistical Connection Admission Control Mechanism for Multiservice IEEE 802.16 Network", In Proceedings of VTC Spring.

[10] R Murali Prasad, 2010. "An Efficient Connection Admission Control Mechanism For IEEE 802.16 Networks", Global Journal of Computer Science and Technology Vol. 10, Issue 8, 97-101.

[11] Sarat Chandra and Anirudha Sahoo, 2007. "An Efficient Call Admission Control for IEEE 802.16 Networks", In Proceedings of the 15th IEEE Workshop on Local \& Metropolitan Area Networks, 188-193.

[12] Lynda Mokdad and Jalel Ben-Othman, 2011. "Admission Control Mechanism and Performance Analysis Based on Stochastic Automata Networks Formalism" J. Parallel Distrib. Comput. 71, 594-602.

[13] Prasun Chowdhury, Iti Saha Misra and Salil K Sanyal, 2010. "An Integrated Call Admission Control and Uplink Packet Scheduling Mechanism for QoS Evaluation of IEEE 802.16 BWA Networks", Canadian Journal on Multimedia and Wireless Networks Vol. 1, No. 3, 14-30.

[14] Romesh Laishram and Iti Saha Misra, 2011. "A Bandwidth Efficient Adaptive Call Admission Control Scheme for QoS Provisioning in IEEE 802.16e Mobile Networks", International Journal of Wireless Information Networks, Vol 18, No. 2, 108-116.

[15] Kalikivayi Suresh, Iti Saha Mishra and Kalpana Saha(Roy), 2008. "Bandwidth and Delay Guaranteed Call Admission Control Scheme for QOS Provisioning in IEEE 802.16e Mobile WiMAX", In Proceedings of IEEE GLOBECOM2008, 1245-1250.

[16] Tzu-Chieh Tsai, Chi-Hong Jiang and Chuang-Yin Wang, 2006. "CAC and Packet Scheduling Using Token Bucket for IEEE 802.16 Networks", Journal of communications, Vol. 1, No. 2, 30-37

[17] Lang Xie, Jie Xiang, 2008. "A Novel Bandwidth Degradation Scheme for Admission Control in IEEE 802.16e Networks", In Proceedings of 4th International Conference on Wireless Communications, Networking and Mobile Computing, WiCOM '08.

[18] Chi-Hong Jiang and Tzu-Chieh Tsai, 2006. "Token Bucket Based CAC and Packet Scheduling for IEEE 802.16 
Broadband Wireless Access Networks", In Proceedings of IEEE CCNC-2006

[19] Sarabjot Singh, Sanjay K. Bose and Maode Ma, 2010. "Analytical and Simulation Studies for Call Admission and Resource Allocation Schemes proposed for WiMAX system", In proceedings of IEEE International Conference on Communication Systems (ICCS), 522 - 526

[20] Iftekhar Ahmad and Daryoush Habibi, 2010. "Call Admission Control Scheme for Improved Quality of Service in WiMAX Communication at Vehicular Speeds", In Proceedings of IEEE GLOBECOM-2010.

[21] D.S.Shu'aibu, S.K.Syed Yusof and N. Fisal, 2010. "Call Admission Control in Mobile WiMAX IEEE802.16e with Dual partitioning", In Proceedings of International
Conference on Intelligent Network and Computing, ICINC2010.

[22] Chadi Tarhini and Tijani Chahed, 2008. "Density-based admission control in IEEE802.16e Mobile WiMAX", In Proceedings of IFIP 1st Wireless Days Conference.

[23] D. S. Shu'aibu and S. K. Syed Yusof, 2011. "Link aware call admission and packet scheduling for best effort and UGS traffics in mobile WiMAX", International Journal of the Physical Sciences Vol. 6, 1694-1701

[24] Loutfi Nuaymi, 2007. WiMAX: Technology for Broadband Wireless Access, John Wiley \& Sons Ltd. 